\title{
Direct Visualization of Soliton CO Overlayers on Supported Pd Nanoparticles
}

Chi M. Yim, Chi L. Pang, Geoff Thornton*

Department of Chemistry and London Centre for Nanotechnology, University College London, 20 Gordon Street, London, WC1H 0AJ, UK

* Corresponding Author. Tel: +44 (0)20 7679 7979, fax: +44 (0)20 7679 0595, email: g.thornton@ucl.ac.uk.

Keywords: carbon monoxide, palladium, nanoparticles, scanning tunneling microscopy 


\section{Abstract}

The interaction of carbon monoxide (CO) molecules with the facets of noble metal nanoparticles forms the basis of many important catalytic reactions. Using scanning tunneling microscopy (STM), we have studied the adsorption of CO molecules on the (111) facets of Pd nanocrystals supported on a rutile $\mathrm{TiO}_{2}(110)$ substrate. We observed four compact $\mathrm{CO}$ overlayers with coverages ranging between 0.5 and 0.6 monolayer. Examination of the positions of the $\mathrm{CO}$ molecules in each of the unit cells reveals that one of the overlayers has a rhombic $(\sqrt{7} \times \sqrt{7}) \mathrm{R} 19.1^{\circ}-4 \mathrm{CO}$ structure. The other three form rectangular structures, namely $(7 \times \sqrt{3})$ rect-8CO, $\mathrm{c}(5 \times \sqrt{3})$ rect $-3 \mathrm{CO}$ and $\mathrm{c}(9 \times \sqrt{3})$ rect $-5 \mathrm{CO}$. These are closely related via a soliton model previously proposed on the basis of infrared absorption spectroscopy and low energy electron diffraction. By imaging the $\mathrm{CO}$ molecules, we provide direct evidence for the soliton model. 


\section{Introduction}

The adsorption of carbon monoxide $(\mathrm{CO})$ on metal surfaces has been widely studied because of its relevance to many technological processes, including methanol synthesis, ${ }^{1}$ the water-gas-shift reaction, ${ }^{2}$ and $\mathrm{CO}$ oxidation in catalytic convertors. ${ }^{3}$ CO molecules typically occupy a number of different adsorption sites and form a variety of ordered overlayers on metal surfaces. Under some conditions, they also cause the surface to restructure, which has important implications for heterogeneous catalysis. $^{4}$

The adsorption behavior of $\mathrm{CO}$ on $\mathrm{Pd}(111)$ is well understood. ${ }^{5-14}$ At low CO coverages $\left(\theta_{\mathrm{CO}}\right), \mathrm{CO}$ molecules occupy threefold fcc-hollow $(H)$ sites, forming a $(\sqrt{3} \times \sqrt{3}) \mathrm{R} 30^{\circ}-1 \mathrm{CO}$ overlayer at 0.33 monolayer (where one monolayer (ML) equals one $\mathrm{CO}$ molecule per primitive $\mathrm{Pd}(111)$ unit cell and where $1 \mathrm{CO}$ indicates that there is one $\mathrm{CO}$ molecule in the primitive unit cell). ${ }^{12}$ Above $0.33 \mathrm{ML}, \mathrm{CO}$ molecules also start to occupy twofold bridge $(B)$ sites in addition to the $H$ sites. At $0.5 \mathrm{ML}$, this leads to the formation of two different $\mathrm{c}(4 \times 2)-2 \mathrm{CO}$ overlayers, one of which has fcc and hep- hollow site occupation $(H H)$, the other of which has bridge site occupation $(B B) .{ }^{12}$ As the CO coverage increases further to between 0.5 and $0.75 \mathrm{ML}$, CO molecules occupy a combination of different sites and form a series of $(n \times \sqrt{3})$, as well as other ordered structures. ${ }^{12-14}$ Finally, at $0.75 \mathrm{ML}$, CO molecules occupy atop ( $T$-), fcc- and hcp- hollow sites, forming $T H-(2 \times 2)-3 \mathrm{CO} .{ }^{12}$

Nanoparticles exhibit properties distinct from their bulk counterparts. ${ }^{3,15,16}$ This, together with their technological relevance, ${ }^{17-19}$ has led to a great deal of research concerning the behavior of supported Pd nanoparticles. ${ }^{20-25}$ Recently, we have investigated the adsorption of $\mathrm{CO}$ on $\mathrm{TiO}_{2}(110)$-supported $\mathrm{Pd}$ nanoparticles. The 
Pd nanoparticles have atomically flat (111) top facets that makes them amenable to study by STM. While some of the CO overlayers observed are identical to their single crystal counterparts, ${ }^{12,25}$ some new CO structures were also observed ${ }^{26}$ and attributed to the strain caused by the 'carpet' growth of Pd nanoparticles across the $\mathrm{TiO}_{2}(110)$ substrate steps.

Here we extend our work on $\mathrm{CO}$ adsorption on Pd nanoparticles to include compact CO phases with coverages of $\theta_{\mathrm{CO}}=0.5-0.6 \mathrm{ML}$. Three of the observed overlayers, directly visualized for the first time, form closely-related rectangular structures. These are the $(7 \times \sqrt{3})$ rect $-8 \mathrm{CO}, \mathrm{c}(5 \times \sqrt{3})$ rect $-3 \mathrm{CO}$, and $\mathrm{c}(9 \times \sqrt{3})$ rect $5 \mathrm{CO}$ structures, whilst the other overlayer has a rhombic $(\sqrt{7} \times \sqrt{7}) \mathrm{R} 19.1^{\circ}-4 \mathrm{CO}$ unit cell.

\section{Experimental}

The experiments were carried out using an Omicron $G m b H$ low temperature STM described in detail elsewhere. ${ }^{25} \mathrm{TiO}_{2}(110)$ samples (PiKem) were cleaned by cycles of $\mathrm{Ar}^{+}$ion sputtering and vacuum annealing to $1000 \mathrm{~K}$. Sample cleanliness and long-range order were checked using X-ray photoelectron spectroscopy (XPS) and low energy electron diffraction (LEED), respectively. Pd was deposited onto the asprepared $\mathrm{TiO}_{2}(110)$ surface with the sample held at $\sim 720 \mathrm{~K}$. The Pd evaporator comprises a Pd wire (Advent, $99.95 \%$ ) wrapped by a W filament that is resistively heated. Depositing $\mathrm{Pd}$ in this way leads to the formation of well-defined $\mathrm{Pd}$ nanocrystals on the $\mathrm{TiO}_{2}(110)$ surface that have (111) top facets. ${ }^{25}$ These nanocrystals have measured average diameters of $26.3 \pm 4.8 \mathrm{~nm}$ and heights of $2.1 \pm 0.4 \mathrm{~nm}$. Due to this relatively large size, we expect their top facets to have properties similar to that 
of the Pd(111) single crystal surface. CO (SIP Analytical Ltd., 99.3\%) was dosed insitu onto the sample at $124 \mathrm{~K}$ via a directional doser that is placed $\sim 100 \mathrm{~mm}$ away from the STM stage. Based on the dosing geometry, the actual CO dose is estimated to be 100 times the nominal dosage as monitored with the ion gauge in the analysis chamber. It is this estimated CO dosage that is reported here.

STM measurements were made in the constant current mode using electrochemically-etched $\mathrm{W}$ tips that were conditioned by outgassing at $\sim 500 \mathrm{~K}$ and by high voltage pulses in STM. All STM images were obtained at $124 \mathrm{~K}$.

\section{Results and Discussions}

Figure 1a shows a STM image recorded from the (111) top facet of a Pd nanocrystal that has an average diameter $(d)$ of $33 \mathrm{~nm}$ and a height $(h)$ of $2.3 \mathrm{~nm}$. Before imaging, the sample was exposed to $11 \mathrm{~L}$ of $\mathrm{CO}\left(1 \mathrm{~L}=1.33 \times 10^{-6}\right.$ mbar.s $)$. This gives rise to the ordered $\mathrm{CO}$ overlayer that can be observed in Fig. 1a. The overlayer is characterized by bright spots that are organized in alternating rows of $\mathrm{W}$ and V shapes. Some of these $\mathrm{W}$ and V shapes are marked in Fig. 1a. The structure can be described with a rectangular unit cell containing eight protrusions. The dimensions of the unit cell are $(1949 \pm 20) \times(476 \pm 20) \mathrm{pm}^{2}$, consistent with a $(7 \times \sqrt{3}) a^{2}$ overlayer, where $a=275 \mathrm{pm}$ is the lattice spacing on the (111) face of Pd.

On this basis, we propose that this overlayer corresponds to a $(7 \times \sqrt{3}) \mathrm{rect}-8 \mathrm{CO}$ structure. 


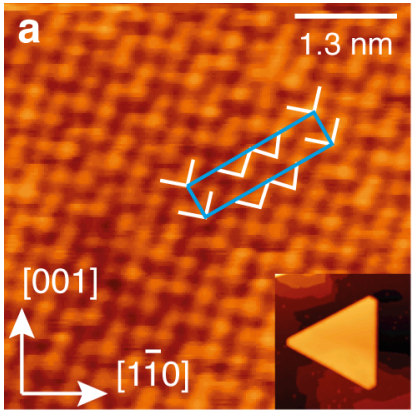

$(5.2 \mathrm{~nm})^{2}, V_{\mathrm{S}}=0.2 \mathrm{~V}, I_{\mathrm{T}}=0.15 \mathrm{nA}$

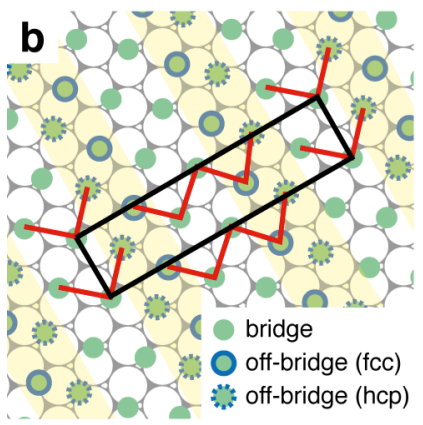

$(7 \times \sqrt{3})$ rect- $8 \mathrm{CO}, \theta_{\mathrm{CO}}=0.571 \mathrm{ML}$

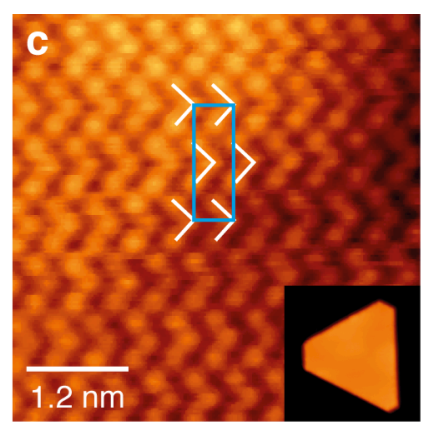

$(4.8 \mathrm{~nm})^{2}, V_{\mathrm{s}}=0.2 \mathrm{~V}, I_{\tau}=0.03 \mathrm{nA}$

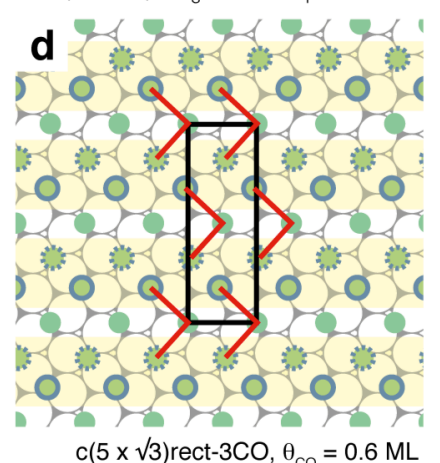

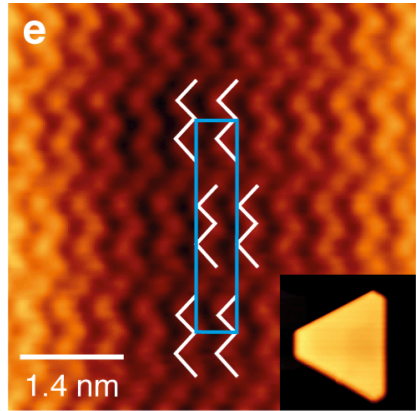

$(5.54 \mathrm{~nm})^{2}, V_{\mathrm{S}}=0.6 \mathrm{~V}, I_{\mathrm{T}}=0.24 \mathrm{nA}$

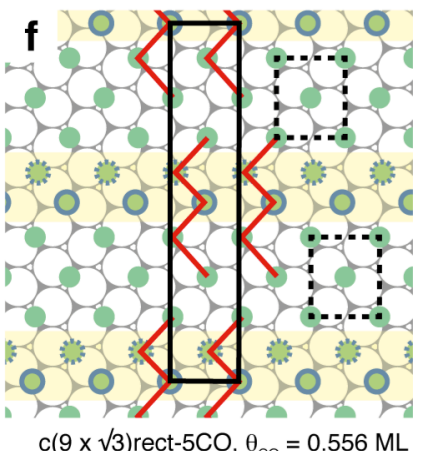

Figure 1. (a,c,e) STM images recorded from the (111) top facets of three different $\mathrm{Pd}$ nanocrystals (insets) supported on the $\mathrm{TiO}_{2}(110)$ surface. The nanocrystals have measured average diameters of $26.3 \pm 4.8 \mathrm{~nm}$ and heights of $2.1 \pm 0.4 \mathrm{~nm}$. The (111) top-facets were covered with different amounts of CO (0.5-0.6 ML), leading to formation of three different $\mathrm{CO}$ overlayers, all of which have $(n \times \sqrt{3})$ rect unit cells: (a) $(7 \times \sqrt{3})$ rect $-8 \mathrm{CO}$, characterized by alternating rows of ' $\mathrm{V}$ ' and ' $\mathrm{W}$ ' shapes (marked by white lines); (c) c $(5 \times \sqrt{3})$ rect $-3 \mathrm{CO}$, characterized by rows of ' $\mathrm{V}$ ' shapes (marked by white lines); (e) $\mathrm{c}(9 \times \sqrt{3})$ rect $-5 \mathrm{CO}$, characterized by rows of ' $\mathrm{W}$ ' shapes (marked by white lines). All STM images were recorded at $124 \mathrm{~K}$. (b,d,f) Corresponding proposed models for the $(n \times \sqrt{3})$ rect overlayers of $\mathrm{CO}$ shown in $(\mathrm{a}, \mathrm{c}, \mathrm{e})$, respectively. Green circles mark the CO molecules that occupy regular bridge sites. Green circles enclosed with solid green lines mark the $\mathrm{CO}$ molecules that occupy off-bridge sites offset towards neighboring fcc hollow sites while those enclosed with dashed lines mark the $\mathrm{CO}$ molecules offset towards the neighboring hcp hollow sites. Domain walls, which are comprised of off-bridge $\mathrm{CO}$ molecules and separate the antiphase domains of $(2 \times \sqrt{3})$ rect $-2 \mathrm{CO}$ are shaded yellow. Black solid lines mark the $(n \times \sqrt{3})$ rect unit cells. Red lines in (b), (d) and (f) indicate the alternating ' $\mathrm{V}$ ' and ' $\mathrm{W}$ ', 'V', and ' $\mathrm{W}$ ' arrangements of CO molecules observed in (a), (c) and (e) respectively. In (f), a $(2 \times \sqrt{3})$ rect $-2 \mathrm{CO}$ unit cell is marked with a dashed line. 
Using infrared absorption spectroscopy (IRAS) and LEED, Tüshaus et al. investigated compact $\mathrm{CO}$ structures on the $\operatorname{Pd}(111)$ single crystal surface, ${ }^{13}$ and proposed a so-called soliton model to account for their experimental results. At the base $\mathrm{CO}$ coverage $\left(\theta_{\mathrm{CO}}\right)$ of $0.5 \mathrm{ML}$, all $\mathrm{CO}$ molecules occupy bridge sites, forming a $(2 \times \sqrt{3})$ rect $-2 \mathrm{CO}$ overlayer. As $\theta_{\mathrm{CO}}$ increases, additional $\mathrm{CO}$ is accommodated in domain walls. While one might expect the $\mathrm{CO}$ molecules inside the domain walls to occupy bridge sites, they are actually offset from the bridge sites in order to minimize intermolecular repulsion, leading to off-bridge occupation. Across the domain walls, anti-phase domains of the existing $(2 \times \sqrt{3})$ rect structures are formed.

Based on Tüshaus et al.'s interpretation, ${ }^{13}$ we propose a similar model to account for the overlayer observed in Fig. 1a. Our model is shown in Fig. 1b and has a unit cell comprising eight $\mathrm{CO}$ molecules: four occupy regular bridge sites (green filled circles), two occupy off-bridge sites offset towards the neighboring fcc-hollow sites (green circles enclosed with solid lines), and the remaining two occupy offbridge sites offset towards the neighboring hcp-hollow sites (green circles enclosed with dashed lines). Together, the $\mathrm{CO}$ molecules at the off-bridge sites form domain walls (shaded in yellow in Fig. 1b) that separate the antiphase domains of the existing bridge-bonded $(2 \times \sqrt{3})$ rect $-2 \mathrm{CO}$. This arrangement of $\mathrm{CO}$ molecules leads to the structure of $(7 \times \sqrt{3})$ rect $-8 \mathrm{CO}\left(\theta_{\mathrm{CO}}=0.571 \mathrm{ML}\right)$. The $\mathrm{W}$ and $\mathrm{V}$ shapes shown in the model are reproduced to scale and superimposed onto the image in Fig. 1a, confirming the good fit with the proposed model.

We observed two other rectangular CO overlayers formed on the (111) topfacets of the Pd nanocrystals. One of the overlayers is characterized by rows of $\mathrm{V}$ 
shapes (Fig. 1c) and has a unit cell containing six bright spots. The unit cell has dimensions of $(1430 \pm 20) \times(480 \pm 20) \mathrm{pm}^{2}$ and is consistent with a $(5 \times \sqrt{3}) a^{2}$ overlayer. The other overlayer is characterized by rows of W shapes (Fig. 1e) and has a unit cell containing ten bright spots. This unit cell has dimensions of $2.44 \times 0.47$ $\mathrm{nm}^{2}$, consistent with a $(9 \times \sqrt{3}) a^{2}$ overlayer.

As before, we construct models for each of these overlayers based on the soliton model. ${ }^{13}$ As shown in Fig $1 \mathrm{~d}$, the model proposed for the $(5 \times \sqrt{3})$ rect overlayer has a unit cell containing six CO molecules: two occupy bridge sites, while the other four occupy off-bridge sites. This leads to a $\mathrm{c}(5 \times \sqrt{3})$ rect $-3 \mathrm{CO}$ structure $\left(\theta_{\mathrm{CO}}=0.6 \mathrm{ML}\right)$. Note that such a model has already been proposed for identical overlayers on $\mathrm{Pd}(111)$ single crystal surfaces. ${ }^{13}$ The model proposed for the $(9 \times \sqrt{3})$ $a^{2}$ overlayer is shown in Fig. 1f and has a unit cell comprising ten CO molecules: six occupy the regular bridge sites while the other four occupy off-bridge sites, leading to a $\mathrm{c}(9 \times \sqrt{3})$ rect $-5 \mathrm{CO}$ structure $\left(\theta_{\mathrm{CO}}=0.556 \mathrm{ML}\right)$. The $\mathrm{V}$ and $\mathrm{W}$ shapes shown in the models of Fig 1c,e are reproduced to scale and superimposed onto the images in Fig. 1c,e thereby confirming the good fit to the models. As with the model in Fig. 1b, the CO molecules at the off-bridge sites are shaded yellow in Fig. 1d,f and form domain walls that separate antiphase domains.

Figure 2 show schematic models for the $(n \times \sqrt{3})$ rect CO structures that have coverages between $3 / 5$ and $7 / 13$ ML. Fig. 2 a shows the $(5 \times \sqrt{3})$ rect structure which comprises anti-phase domain walls (highlighted in yellow) that alternate with domains that consist of single rows of $\mathrm{CO}$ molecules in bridge sites (marked with 
green circles). Each domain wall contains two rows of $\mathrm{CO}$ molecules. To minimize intermolecular repulsion, ${ }^{13}$ these are displaced away from each other towards the neighboring threefold sites, leading to off-bridge occupation. For the $(5 \times \sqrt{3})$ rect structure shown in Fig. 2a, molecules in one of the CO rows (marked with green circles enclosed with blue solid lines) are offset to the bottom-left, i.e. towards the neighboring fcc-hollow sites, whilst the other row contains $\mathrm{CO}$ molecules (marked with green circles with blue dashed lines) offset in the opposite direction towards the top-right, i.e. towards the neighboring hep-hollow sites . The displacement directions of the $\mathrm{CO}$ molecules in the domain walls are indicated with arrows in Fig. 2a(left). We will refer to domain walls with this arrangement of CO molecules as type $A$. 


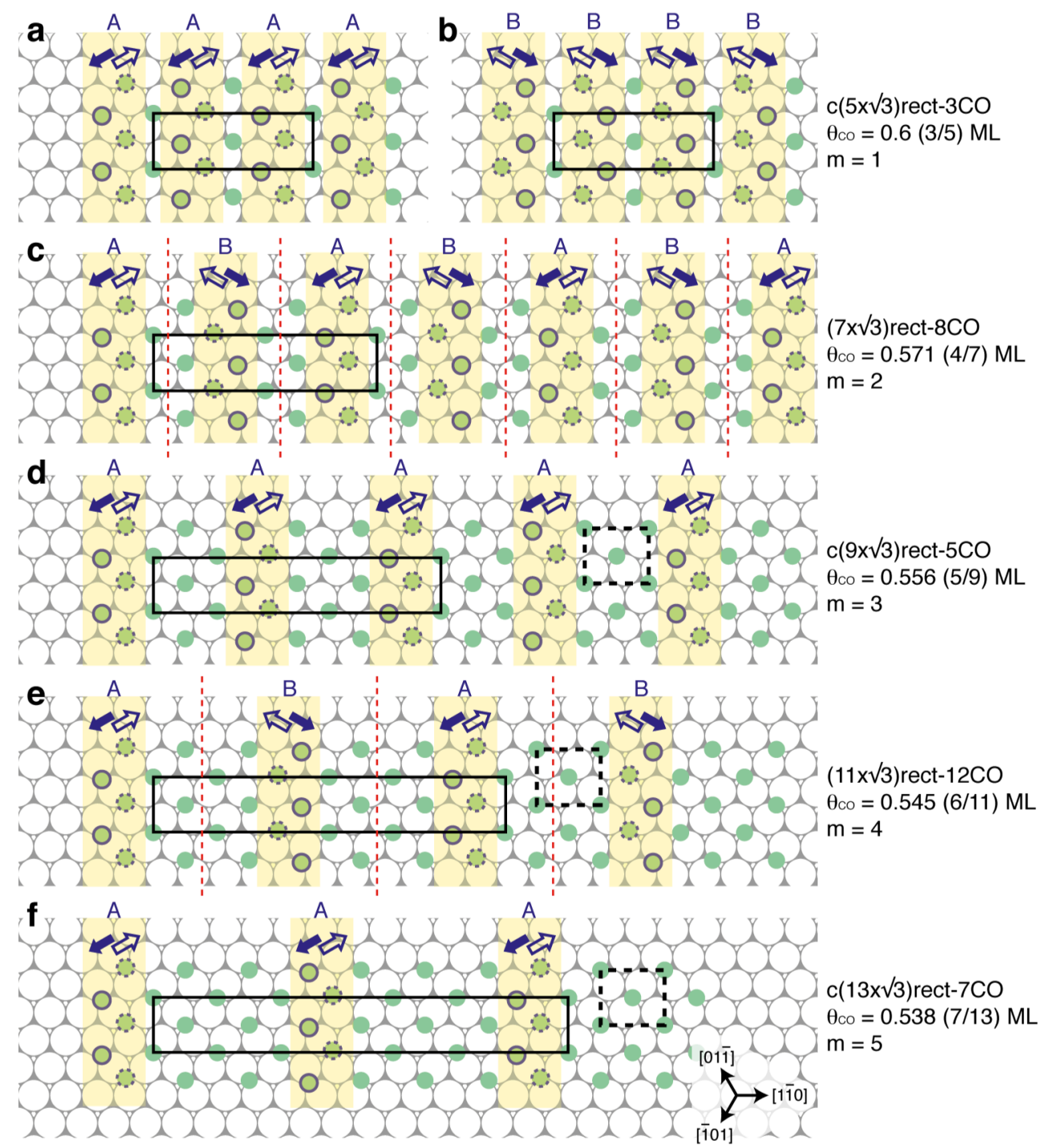

Figure 2. Schematic illustrations of the $(n \times \sqrt{3})$ rect structures on $\operatorname{Pd}(111)$ with $\mathrm{CO}$ coverages between 0.6 and $0.538 \mathrm{ML}$. Green circles mark the $\mathrm{CO}$ molecules that occupy bridge site in the $(2 \times \sqrt{3})$ rect domains. Yellow shading marks the anti-phase domain walls. Inside the domain walls, green circles enclosed with blue solid lines mark the off-bridge $\mathrm{CO}$ molecules that are offset towards the neighboring fcc-hollow sites, while those enclosed with blue dashed lines mark the off-bridge $\mathrm{CO}$ molecules that are offset towards the neighboring hcp-hollow sites. Filled (open) arrows indicate the displacement directions of the off-bridge $\mathrm{CO}$ molecules that are offset towards the neighboring fcc- (hcp-) hollow sites, respectively. The difference in their displacement directions leads to two different types of domain walls that we denote ' $A$ ' and ' $B$ '. Black solid lines indicate the $(n \times \sqrt{3})$ rect unit cells whereas black dashed lines mark some of the $(2 \times \sqrt{3})$ rect unit cells. The $\mathrm{CO}$ coverage $\left(\theta_{\mathrm{CO}}\right)$ and the number of $\mathrm{CO}$ rows, $m$, in each of the $(2 \times \sqrt{3})$ rect domains are indicated. In (c) and (e), red dashed lines mark glide reflection planes formed between $\mathrm{CO}$ patches with domain walls of different types. 
Figure $2 \mathrm{~b}$ shows another $(5 \times \sqrt{3})$ rect structure that has a different arrangement of $\mathrm{CO}$ molecules inside its domain walls that we denote as type $B$. It consists of two rows of off-bridge $\mathrm{CO}$, one of which contains $\mathrm{CO}$ molecules offset to the top-left, i.e. towards the neighboring hcp-hollow sites, the other containing $\mathrm{CO}$ molecules offset to the bottom-right, i.e. towards the neighboring fcc-hollow sites. The two models in Fig. 2a,b cannot be distinguished from the STM images (Fig. 1).

Figure 2c shows a model of the $(7 \times \sqrt{3})$ rect structure. It contains $(2 \times \sqrt{3})$ rect domains that are separated alternately by type $A$, then type $B$ domain walls. In Fig. 2d-f, as we continue to increase the value of $n$ in the $(n \times \sqrt{3})$ rect structures, it becomes apparent that there is a pattern to the type of domain wall observed. When there are an odd number of $\mathrm{CO}$ rows in the antiphase domains, the domain walls are all of either type $A$ or type $B$, whereas with an even number of CO rows, the domain walls alternate between $A$ and $B$. As indicated by the red dashed lines in Fig. 2c, e, the $\mathrm{CO}$ arrangement in the latter case also gives rise to glidereflection symmetry between $\mathrm{CO}$ patches containing domain walls of different types.

If we denote the number of rows in the $(2 \times \sqrt{3})$ rect domains as an integer variable, $m$, we can also express a number of other relationships. The length of the soliton $(n \times \sqrt{3})$ rect unit cell along $\{\overline{1} 01\}$, is given by $n a$, where $n=2 m+3$ and the number of CO molecules present in the $(n \times \sqrt{3})$ rect unit cell is $(2 m+4)$ which gives a CO coverage of $(m+2) /(2 m+3) \mathrm{ML}$.

Exposing the $\mathrm{c}(9 \times \sqrt{3})$ rect $-5 \mathrm{CO}$ overlayer (Fig. 1c) to an extra $0.33 \mathrm{~L} \mathrm{CO}$ leads to a structural conversion into another compact CO structure. As shown in the 
STM image of Fig. 3a, the newly-formed structure is characterized by a rhombic arrangement of $\mathrm{CO}$ and has around a quarter of the $\mathrm{CO}$ molecules appearing brighter than the remainder. The white rhombus in Fig. 3a is drawn with the vertices over the brightest spots and measurements reveal the side lengths to be $718 \pm 10 \mathrm{pm}$, very close to the value of $\sqrt{ } 7 a$. Furthermore, one of its sides is aligned at an angle of $19.1^{\circ}$ from the vertical direction (marked by the blue guideline). As such, we propose a $(\sqrt{7} \times \sqrt{7}) R 19.1^{\circ}$ unit cell for this overlayer, although we note that the ordering is not perfect. For instance, the green rhombus only has bright spots at two of its vertices. A $(\sqrt{7} \times \sqrt{7}) R 19.1^{\circ}$ structure has been reported on several single crystal metal surfaces: $\operatorname{Pd}(111),{ }^{12} \mathrm{Rh}(111),{ }^{27}$ and $\mathrm{Ni}(111) .{ }^{28}$ On Ni(111), the $(\sqrt{7} \times \sqrt{7}) R 19.1^{\circ}-4 \mathrm{CO}$ unit cell has one $\mathrm{CO}$ occupying an atop site and the remaining three $\mathrm{CO}$ molecules in bridge sites. $^{28}$ On this basis, we propose our $(\sqrt{7} \times \sqrt{7}) R 19.1^{\circ}$ structure in Fig. 3a to have the same occupancy, as illustrated by the model in Fig. 3c. To further confirm our assignment of the structure, we constructed a $\operatorname{Pd}(111)$ lattice at the center of the STM image (Fig. 3b) based on the assumption that the brightest spots are $\mathrm{CO}$ molecules at atop sites. Examination of the positions of the STM spots with respect to the $\operatorname{Pd}(111)$ grid reveals that all other $\mathrm{CO}$ molecules in the rhombus (darker STM spots) occupy bridge sites, hence confirming a $(\sqrt{7} \times \sqrt{7}) R 19.1^{\circ}-4 \mathrm{CO}$ overlayer with atop and bridge site occupation.

We note that the $(\sqrt{7} \times \sqrt{7}) R 19.1^{\circ}-4 \mathrm{CO}$ overlayer has the same coverage as the $(7 \times \sqrt{3})$ rect $-8 \mathrm{CO}$ overlayer. In the $(7 \times \sqrt{3})$ rect $-8 \mathrm{CO}$ overlayer, half of the $\mathrm{CO}$ molecules occupy regular bridge sites while the other half occupy off-bridge sites. In 
contrast, the $(\sqrt{7} \times \sqrt{7}) R 19.1^{\circ}-4 \mathrm{CO}$ structure has $3 / 4$ of $\mathrm{CO}$ molecules occupying regular bridge sites with the remaining $1 / 4$ of $\mathrm{CO}$ molecules occupying atop sites. Presumably, the decrease in stability in having two out of every four CO molecules in off-bridge sites is similar to that incurred by accommodating one out of every four CO molecules in atop sites. 


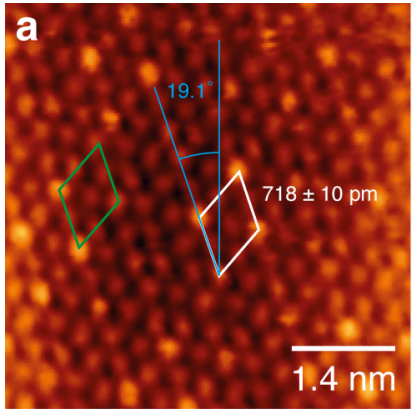

$(5.54 \mathrm{~nm})^{2}, V_{\mathrm{S}}=0.6 \mathrm{~V}, \mathrm{I}_{\mathrm{T}}=0.24 \mathrm{nA}$
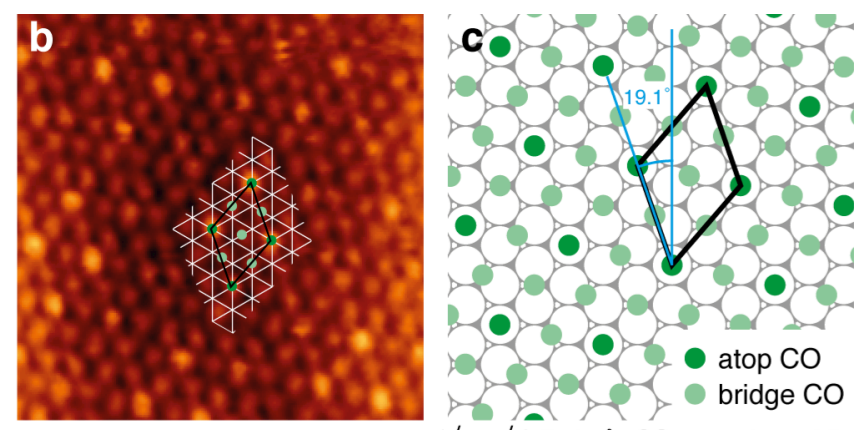

$(\sqrt{7} \times \sqrt{7})-\mathrm{R} 19.1^{\circ}-4 \mathrm{CO}, \theta_{\mathrm{CO}}=0.571 \mathrm{ML}$

Figure 3. (a) STM image of $(\sqrt{7} \times \sqrt{7}) R 19.1^{\circ}-4 \mathrm{CO}$ structure following an additional exposure of $0.33 \mathrm{~L} \mathrm{CO}$ to the same island as shown in the inset of Fig. 1c. A rhombic structure is marked with white lines and is formed by four brighter spots at each of the vertices. Green lines mark a rhombus structure that has only two brighter spots at its vertices. The rhombic unit cell has a side-length of $718 \pm$ $10 \mathrm{~nm}$, very close to the value of $\sqrt{7 a}$ (where $\mathrm{a}=275 \mathrm{pm}$ is the interatomic distance on a $\operatorname{Pd}(111)$ surface). The light blue guideline is aligned to one side of the rhombic unit cell and is exactly $19.1^{\circ}$ from the vertical, as would be expected from the ideal $(\sqrt{7} \times \sqrt{7}) R 19.1^{\circ}$ structure. The STM image was recorded at 124 K. (b) As (a). On the basis that the brightest spots correspond to CO molecules at atop sites, a perfect hexagonal $\operatorname{Pd}(111)$ grid is superimposed over part of the image, revealing a $(\sqrt{7} \times \sqrt{7}) R 19.1^{\circ}$ unit cell with atop and bridge site occupation.

(c) Proposed model for the $(\sqrt{7} \times \sqrt{7}) R 19.1^{\circ}-4 \mathrm{CO}$ unit cell. Dark and light green circles mark the $\mathrm{CO}$ molecules that occupy atop and bridge sites, respectively.

\section{Summary}

In conclusion, using STM we have observed four compact overlayers of $\mathrm{CO}$ that form on the (111) top-facets of $\mathrm{TiO}_{2}(110)$-supported $\mathrm{Pd}$ nanocrystals. The $\mathrm{CO}$ coverages of these overlayers range between 0.5 and 0.6 ML. Three overlayers were observed with rectangular unit cells: $(7 \times \sqrt{3})$ rect- $8 \mathrm{CO}, \mathrm{c}(5 \times \sqrt{3})$ rect $-3 \mathrm{CO}$, and $\mathrm{c}(9 \times \sqrt{3})$ rect $-5 \mathrm{CO}$. The models proposed for these phases are all related to the soliton model suggested by Tüshaus et al. ${ }^{13} \mathrm{CO}$ molecules occupy off-bridge sites in domain walls that separate $\mathrm{CO}$ in bridge sites that form anti-phase domains. The 
other phase observed has a rhombic unit cell, $(\sqrt{7} \times \sqrt{7}) R 19.1^{\circ}-4 \mathrm{CO}$, with $\mathrm{CO}$ molecules occupying atop and bridge sites.

\section{Acknowledgements}

This work was supported by the European Cooperation in Science and Technology Action CM1104, the European Research Council Advanced Grant ENERGYSURF (GT), the Royal Society (UK), and Alexander von Humboldt Stiftung (Germany). 
References

(1) Behrens, M.; Studt, F.; Kasatkin, I.; Kühl, S.; Hävecker, M.; Abild-Pedersen, F.; Zander, S.; Girgsdies, F.; Kurr, P.; Kniep, B.-L.; et al. The Active Site of Methanol Synthesis over $\mathrm{Cu} / \mathrm{ZnO} / \mathrm{Al}_{2} \mathrm{O}_{3}$ Industrial Catalysts. Science 2012, $336,893-897$.

(2) Fu, Q; Saltsburg, H; Flytzani-Stephanopoulos, M. Active Nonmetallic Au and Pt Species on Ceria-Based Water-Gas Shift Catalysts. Science 2003, 301, 935-938.

(3) Jackson, S. D.; Hargreaves, J. S. J. Metal Oxide Catalysis; Wiley: WeinHeim, 2008.

(4) Tao, F.; Dag, S.; Wang, L.-W.; Liu, Z.; Butcher, D. R.; Bluhm, H.; Salmeron, M.; Somorjai, G. A. Break-Up of Stepped Platinum Catalyst Surfaces by High CO Coverage. Science 2010, 327, 850-853.

(5) Loffreda, D.; Simon, D.; Sautet, P. Dependence of Stretching Frequency on Surface Coverage and Adsorbate-Adsorbate Interactions: a DensityFunctional Theory Approach of CO on Pd(111). Surf. Sci. 1999, 425, 68-80.

(6) Miranda, R.; Wandelt, K.; Rieger, D.; Schnell, R. D. Angle-Resolved Photoemission of CO Chemisorption on Pd(111). Surf. Sci. 1984, 139, 430 442.

(7) Ohtani, H.; Van Hove, M. A.; Somorjai, G. A. LEED Intensity Analysis of the Surface-Structures of $\operatorname{Pd}(111)$ and of $\mathrm{CO}$ Adsorbed on $\operatorname{Pd}(111)$ in a $(\sqrt{ } 3 \times \sqrt{ } 3)$ R30 Arrangement. Surf. Sci. 1987, 187, 372-386.

(8) Gießel, T.; Schaff, O.; Hirschmugl, C. J.; Fernandez, V.; Schindler, K.-M.; Theobald, A.; Bao, S.; Lindsay, R.; Berndt, W.; Bradshaw, A. M. A Photoelectron Diffraction Study of Ordered Structures in the Chemisorption 
System Pd $\{111\}$-CO. Surf. Sci. 1998, 406, 90-102.

(9) Kuhn, W. K.; Szanyi, J.; Goodman, D. W. CO Adsorption on Pd(111): the Effects of Temperature and Pressure. Surf. Sci. 1992, 274, L611-L618.

(10) Ozensoy, E.; Meier, D. C.; Goodman, D. W. Polarization Modulation Infrared Reflection Absorption Spectroscopy at Elevated Pressures: CO Adsorption on $\operatorname{Pd}(111)$ at Atmospheric Pressures. J. Phys. Chem. B 2002, 106, 9367-9371.

(11) Surnev, S.; Sock, M.; Ramsey, M. G.; Netzer, F. P.; Wiklund, M.; Borg, M.; Andersen, J. N. CO Adsorption on Pd(111): a High-Resolution Core Level Photoemission and Electron Energy Loss Spectroscopy Study. Surf. Sci. 2000, 470, 171-185.

(12) Rose, M. K.; Mitsui, T.; Dunphy, J.; Borg, A.; Ogletree, D. F.; Salmeron, M.; Sautet, P. Ordered Structures of CO on Pd (111) Studied by STM. Surf. Sci. 2002, 512, 48-60.

(13) Tüshaus, M.; Berndt, W.; Conrad, H.; Bradshaw, A. M.; Persson, B. Understanding the Structure of High Coverage CO Adlayers. Appl. Phys. A 1990, 51, 91-98.

(14) Morkel, M.; Unterhalt, H.; Salmeron, M.; Rupprechter, G.; Freund, H.-J. SFG Spectroscopy from $10^{-8}$ to 1000 Mbar: Less-Ordered CO Structures and Coadsorption on Pd. Surf. Sci. 2003, 532-535, 103-107.

(15) Zhdanov, V. P.; Kasemo, B. Simulations of the Reaction Kinetics on Nanometer Supported Catalyst Particles. Surf. Sci. Rep. 2000, 39, 25-104.

(16) Tauster, S. J. Strong Metal-Support Interactions. Acc. Chem. Res. 1987, 20, 389-394.

(17) Gao, W.; Chen, J.; Guan, X.; Jin, R.; Zhang, F.; Guan, N. Catalytic Reduction 
of Nitrite Ions in Drinking Water Over $\mathrm{Pd}-\mathrm{Cu} / \mathrm{TiO}_{2}$ Bimetallic Catalyst. Catal. Today 2004, 93-95, 333-339.

(18) Kim, W. J.; Kang, J. H.; Ahn, I. Y.; Moon, S. H. Deactivation Behavior of a $\mathrm{TiO}_{2}$-Added Pd Catalyst in Acetylene Hydrogenation. J. Catal. 2004, 226, $226-229$.

(19) Imagawa, H.; Tanaka, T.; Takahashi, N.; Matsunaga, S.; Suda, A.; Shinjoh, H. Synthesis and Characterization of $\mathrm{Al}_{2} \mathrm{O}_{3}$ And $\mathrm{ZrO}_{2}-\mathrm{TiO}_{2}$ Nano-Composite as a Support for $\mathrm{NO}_{\mathrm{X}}$ Storage-Reduction Catalyst. J. Catal. 2007, 251, 315320.

(20) Bowker, M.; Stone, P.; Bennett, R.; Perkins, N. CO Adsorption on a $\mathrm{Pd} / \mathrm{TiO}_{2}$ (110) Model Catalyst. Surf. Sci. 2002, 497, 155-165.

(21) Rupprechter, G. Sum Frequency Generation and Polarization-Modulation Infrared Reflection Absorption Spectroscopy of Functioning Model Catalysts From Ultrahigh Vacuum to Ambient Pressure. Adv. Catal. 2007, 51, 133263.

(22) Fischer-Wolfarth, J.-H.; Farmer, J. A.; Flores-Camacho, J. M.; Genest, A.; Yudanov, I. V.; Rösch, N.; Campbell, C. T.; Schauermann, S.; Freund, H.-J. Particle-Size Dependent Heats of Adsorption of CO on Supported Pd Nanoparticles as Measured with a Single-Crystal Microcalorimeter. Phys. Rev. B 2010, 81, 241416.

(23) Peter, M.; Adamovsky, S.; Flores Camacho, J. M.; Schauermann, S. Energetics of Elementary Reaction Steps Relevant for CO Oxidation: CO and $\mathrm{O}_{2}$ Adsorption on Model Pd Nanoparticles and Pd(111). Faraday Discuss. 2013, 162, 341-354.

(24) Peter, M.; Flores Camacho, J. M.; Adamovski, S.; Ono, L. K.; Dostert, K.-H.; 
O'Brien, C. P.; Roldan Cuenya, B.; Schauermann, S.; Freund, H.-J. Trends in der Bindungsstärke von Oberflächenspezies auf Nanopartikeln: Wie verändert sich die Adsorptionsenergie mit der Partikelgröße? Angew. Chem. 2013, 125, 5282-5287.

(25) Yim, C. M.; Pang, C. L.; Humphrey, D. S.; Muryn, C. A.; Schulte, K.; Pérez, R.; Thornton, G. CO and O Overlayers on Pd Nanocrystals Supported on $\mathrm{TiO}_{2}(110)$. Faraday Discuss. 2013, 162, 191-200.

(26) Yim, C. M.; Pang, C. L.; Hermoso, D. R.; Dover, C. M.; Muryn, C. A.; Maccherozzi, F.; Dhesi, S. S.; Pérez, R.; Thornton, G. Influence of Support Morphology on the Bonding of Molecules to Nanoparticles. Proc. Natl. Acad. Sci. U.S.A. 2015, 112, 7903-7908.

(27) Cernota, P.; Rider, K.; Yoon, H. A.; Salmeron, M.; Somorjai, G. Dense Structures Formed by $\mathrm{CO}$ on $\mathrm{Rh}(111)$ Studied by Scanning Tunneling Microscopy. Surf. Sci. 2000, 445, 249-255.

(28) Biberrian, J. P.; Van Hove, M. A. A New Model for CO Ordering at High Coverages on Low Index Metal Surfaces: a Correlation between LEED, HREELS and IRS: II. CO Adsorbed on Fcc (111) and Hcp (0001) Surfaces. Surf. Sci. 1984, 138, 361-389. 
Table of Contents Image
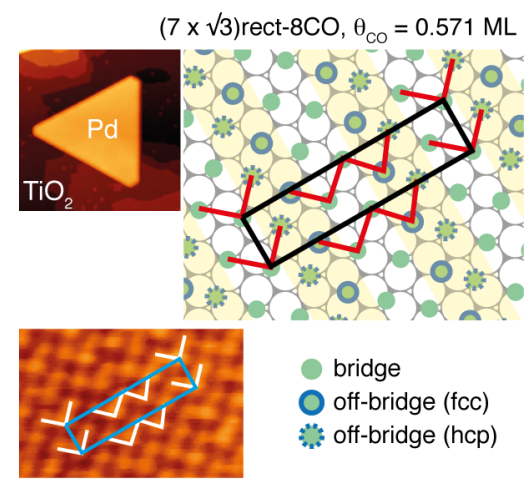

bridge

off-bridge (fcc)

off-bridge (hcp)

$\mathrm{CO} / \mathrm{Pd}(111)$ facet

2 inches by 2 inches 